\title{
Classifying the provably total functions of PA*
}

\author{
Andreas Weiermann \\ Mathematical Institute \\ P.O. Box 80010 \\ 3508 TA Utrecht \\ The Netherlands \\ e-mail: weierman@math.uu.nl
}

\begin{abstract}
We give a self-contained and streamlined version of the classification of the provably computable functions of PA. The emphasis is put on illuminating as good as seems possible the intrinsic computational character of the standard cut elimination process. The article is intended to be suitable for teaching purposes and just requires basic familiarity with PA and the ordinals below $\varepsilon_{0}$. (Familiarity with a cut elimination theorem for a Gentzen or Tait calculus is helpful but not presupposed).
\end{abstract}

\section{Introduction}

In this article we reprove a classical result on the classification of the provably recursive functions of first order Peano arithmetic. Our main goal is to provide a proof which explains as good as seems possible the computational complexity behind standard cut elimination procedures. The presentation is intended to prove useful for teaching purposes. In particular it offers a quick and self contained way to various combinatorial independence results.

The classification result for the provably recursive functions of Peano arithmetic goes back to Kreisel (1952) [14] (with roots going back to Ackermann (1940) [1]): Roughly speaking the result is that the class of provably recursive functions of Peano arithmetic is exactly the class of functions (which are primitive recursive in functions) which can be defined by recursion along initial segments of $\varepsilon_{0}$.

Folklore (proof-theoretic) proofs for this result [cf., for example, Schwichtenberg (1977) [19], Takeuti (1987) [21], Buchholz (1991) [4] or Friedman and Sheard (1995) [11] for such proofs] rely on non trivial metamathematical evaluations of the Gentzen- or Schütte-style proof-theoretic analyses of Peano arithmetic. A

* Research supported by a Heisenberg-Fellowship of the Deutsche Forschungsgemeinschaft 
proof of the classification result that does not rely on metamathematical considerations - like primitive recursive stipulations of codes of infinite proof-trees - has been given in [Buchholz and Wainer (1987) [7] and Fairtlough and Wainer (1998) [10]]. Other proofs for the classification result which are based on non standard models can be found, for example, in [Hájek and Pudlák (1993) [13] or Avigad and Sommer (1997) [2]]. This paper aims at a solution to the following problem:

Give a proof for the classification result which is conceptually and technically as smooth as seems possible!

This has been solved in our opinion to some extent already in [3] where certain operators (which we call Skolem operators) have been introduced for controlling witness information in derivations. In this paper we generalize the approach from [3] to rather general Skolem operators which are only required to be weakly increasing (i.e. $x \leq y \Rightarrow F(x) \leq F(y)$ ) and inflationary (i.e. $x \leq F(x)$ for every $x \in \mathbb{N}$.) In contrast to [3] this paper does not depend on the approach to hierarchies found in [8].

We include a result on majorizing operators by standard Hardy functions. This rounds off the result since the Hardy functions of level below a fixed $\alpha<\varepsilon_{0}$ are easily shown to be computable within PA. The short presentation makes it possible to teach the whole classification result within two double hours in an introductory course in logic (after having treated provable instances of transfinite induction in PA). This might be a good starting point to study combinatorial independence results in more detail starting with

$$
\mathrm{PA} \nvdash \forall x \exists y H_{\varepsilon_{0}}(x)=y
$$

as a basis for the unprovability result for hydra games or Goodstein sequences ${ }^{1}$.

It is particular illuminating to study the transition of the Skolem operators during the cut elimination process. We introduce basically two operations on operators. The $R$ operator used in the cut reduction lemma is more or less the composition functional. For carrying out the cut reduction theorem we define a suitable transfinite iteration functional $R^{\alpha}$ which gives the $\alpha$-th iterate of $R$ when applied to $F$. Then the $\alpha$-th iterated application of the reduction lemma in the proof of the reduction theorem for a derivation of height bounded by $\alpha$ is reflected on the side of operators by an application of $R^{\alpha}$.

This paper is essentially self contained. Missing details on ordinals below $\varepsilon_{0}=\min \left\{\xi: \xi=\omega^{\xi}\right\}$ can be taken, for example, from Schütte's textbook [18] or, for example, from the masterpieces provided by Buchholz on his home-page.

This paper profits from previous work of Buchholz in at least three ways. Firstly, on the technical side we modeled our infinitary system along the lines devised in one of Buchholz lecture notes. (Other choices would have been convenient here as well.) Secondly we profited from Buchholz's for reaching method of operator controlled derivations which is to our best knowledge today's most

\footnotetext{
${ }^{1}$ Indeed, this paper emerged from a lecture given by the author on recursion and proof theory at Utrecht University. The author is grateful for Ieke Moerdijk for the corresponding arrangements.
} 
powerful framework for the proof theory of strong systems. In this paper we show how to miniaturize the operator method for studying provably recursive functions in an illuminating and smooth way. Thirdly we have employed in the proof of Theorem 4 methods which are implicit in a joint paper with Buchholz and Cichon [8].

\section{The computational complexity of cut elimina- tion in the infinitary calculus $Z^{\infty}$}

We work with an infinitary Gentzen style calculus $Z^{\infty}$. Its first characteristic feature is the so called $\omega$ rule, which allows to deduce $\forall x A(x)$ from infinitely many premises $A(n)(n \in \mathbb{N})$. Using this inference rule one can derive all instances of the induction schema so that PA derivations can be turned into $Z^{\infty}$ derivations. Since $Z^{\infty}$ has no axioms for induction the cut elimination technique for predicate calculus can be extended without problems to it yielding cut free $Z^{\infty}$ derivations.

It is well known that Herbrand's theorem on $\exists$-inversion follows as a corollary from Gentzen's cut elimination theorem. Unfortunately this approach does not extend immediately to $Z^{\infty}$. The second characteristic feature of our calculus $Z^{\infty}$ is the use of a special bookkeeping formalism (which we call Skolem operators) on existential witnesses so that we can mimic the proof of Herbrand's theorem for cut free derivations. For getting the desired classification result we only have to guarantee that the bookkeeping behaves well during the cut elimination process. This is particularly illuminating since we can learn how cut elimination successively leads to stronger and stronger operators.

To fix the context let us agree to work with logical symbols $\forall, \rightarrow$ and $\perp$ from which all other logical symbols are defined as usual. There is only one relation symbol, =, for equality, and there are function symbols for each primitive recursive function. These function symbols include in particular a constant 0 for zero and a unary function symbol $S$ for the successor function. The resulting language is denoted by $L_{Z}$. In the sequel it will prove useful having extended the language of PA to the language of such a conservative extension $Z$ containing the function symbols and defining equations for the primitive recursive functions. The advantage is that Kleene's $T$ predicate can be handled via an atomic formula of lowest possible logical complexity. Otherwise we would have to deal with bounded quantifiers in the collapsing theorem, which although possible, would create extra complications.

In the context of $Z^{\infty}$ Greek capital letters like $\Gamma, \Delta$ stand for finite sets of closed $L_{Z}$ formulas (i.e. formulas having no free variables). We write $\Gamma \supset C$ for the sequent with antecedent formulas from $\Gamma$ and succedent $C$. The intended meaning of a sequent $\Gamma \supset C$ is that the conjunction over all members of the set of antecedent formulas implies the succedent formula $C$. For each closed term $t$ in $L_{Z}$ we have a standard interpretation $\operatorname{val}(t)$ called the value of $t$. In particular we have for each non negative integer a standard numeral $\underline{n}$ such that 
$\operatorname{val}(\underline{n})=n$.

For each formula $C$ in the language $L_{Z}$ we define its rank $r k(C)$ measuring its logical complexity as follows:

1. $r k(C)=0$ if $C$ is atomic.

2. $r k(A \rightarrow B)=\max \{r k(A), r k(B)\}+1$

3. $r k(\forall x A)=r k(A)+1$.

We are going to introduce a notion $F \vdash_{m}^{\alpha} \Gamma \supset C$ where the function $F$ : $\mathbb{N} \rightarrow \mathbb{N}$ controls the computational content of the derivation of $\Gamma \supset C$, where $\alpha$ is a bound for the depth of the derivation and $m$ is a bound for the ranks of cut formulas. The calculus is designed after the truth definition for the standard model for the Peano axioms. Thus if $\Gamma \supset C$ has been derived, then $\mathbb{N} \models \bigwedge_{D \in \Gamma} D \rightarrow C$ or equivalently

$$
\mathbb{N} \models\left(\bigvee_{D \in \Gamma} \neg D\right) \vee C .
$$

The new ingredient in the derivations is the operator control via functions $F: \mathbb{N} \rightarrow \mathbb{N}$. The underlying idea for their use is quite simple. In case of an existential introduction $A(\underline{k}) \vdash \exists x A(x)$ one keeps track on the witness $k$ by demanding $k \leq F(0)$. This has the effect that a cut free derivation $F \vdash \exists x A(x)$ yields that there is a $k \leq F(0)$ such that $\mathbb{N} \models A[k]$. The relation $F \vdash$ is then set up so that this crucial information on witnesses is conserved during derivations. For controlling applications of the $\omega$-rule we demand that $F \vdash \forall x A(x)$ follows from $F[n] \vdash A(\underline{n})$ where $F[n]$ is the operator resulting from $F$ by feeding in information on $n$. ( $F[n]$ will be defined precisely soon.) It will be easy to embed the formal system PA into the infinitary system $Z^{\infty}$.

The critical step in the analysis will be the study of the transformation of the operators during the cut elimination. What is nice about this approach is (as already said) that the cut reduction lemma produces compositions of the control functions (reminiscent of the Brouwer Heyting Kolmogorov interpretation). Moreover the cut elimination theorem gives rise to iterated applications of the cut reduction lemma and hence to a transfinite iteration of the composition operator resulting from the cut elimination lemma. We show that this can be modeled smoothly in terms of pure control operators. In carrying out this outline it turns out to be necessary in formulating $F \vdash_{m}^{\alpha}$ that in each step the number-theoretic content of $\alpha$ is contained in the operator $F$ via a condition like $m c(\alpha) \leq F(0)$ where $m c(\alpha)$ is the largest natural number occurring in the Cantor normal form of $\alpha$.

Recall that a function $F: \mathbb{N} \rightarrow \mathbb{N}$ is called weakly increasing if $x \leq y$ implies $F(x) \leq F(y)$ for all $x, y \in \mathbb{N}$ and $F$ is called inflationary if $x \leq F(x)$ for all $x \in \mathbb{N}$. We call a function $F$ Skolem operator if it is weakly increasing and inflationary function. We write $F \leq G$ iff $F(x) \leq G(x)$ for all $x \in \mathbb{N}$. We write $F[m]$ for $l \mapsto F(\max \{l, m\})$. Then $F[m]$ is Skolem operator if $F$ is. Moreover, if $F$ and $G$ are Skolem operators, then $F \circ G$ and $F+G$ are it, too. 
As a measure for the number-theoretic content of an ordinal $\alpha$ we take the maximal natural number which shows up in the Cantor normal form of $\alpha$. Thus $m c(0):=0$ and for $\alpha=\omega^{\alpha_{1}} \cdot k_{1}+\cdots+\omega^{\alpha_{n}} \cdot k_{n}>0$ with $\alpha_{1}>\ldots>\alpha_{n}$ and $k_{1}, \ldots k_{n}>0$ let

$$
m c(\alpha):=\max \left\{k_{1}, \ldots, k_{n}, m c\left(\alpha_{1}\right), \ldots, m c\left(\alpha_{n}\right)\right\} .
$$

Then for any $\alpha<\varepsilon_{0}$ and $d<\omega$ the number of $\xi<\alpha$ such that $m c(\xi) \leq d$ is always finite.

We call a formula $A$ a literal if $r k(A)=0$ or $A=\neg C$ and $r k(C)=0$.

After these preliminaries let us define

$$
F \vdash_{m}^{\alpha} \Gamma \supset C
$$

iff $m c(\alpha) \leq F(0)$ and one of the following cases holds. (The underlying semantic of these rules should be clear from (1).)

1. $(C$ is a literal $\& \mathbb{N} \models C)$ or $(\exists D \in \Gamma)[D$ is a literal $\& \mathbb{N} \models \neg D]$,

2. $C=A \rightarrow B \& F \vdash_{m}^{\alpha_{0}} A, \Gamma \supset B \& \alpha_{0}<\alpha$,

3. $C=\forall x A(x) \&(\forall n)\left[F[n] \vdash_{m}^{\alpha_{n}} \Gamma \supset A(\underline{n}) \& \alpha_{n}<\alpha\right]$,

4. $A \rightarrow B \in \Gamma \& F \vdash_{m}^{\alpha_{0}} \Gamma \supset A \& F \vdash_{m}^{\alpha_{0}} B, \Gamma \supset C \& \alpha_{0}<\alpha$,

5. $\forall x A(x) \in \Gamma \& F \vdash_{m}^{\alpha_{0}} A(\underline{k}), \Gamma \supset C \& k \leq F(0) \& \alpha_{0}<\alpha$,

6. $r k(D)<m \& F \vdash_{m}^{\alpha_{0}} \Gamma \supset D \& F \vdash_{m}^{\alpha_{0}} \Gamma, D \supset C \& \alpha_{0}<\alpha$,

7. $C$ atomic \& $F \vdash_{m}^{\alpha_{0}} \neg C, \Gamma \supset \perp \& \alpha_{0}<\alpha$.

We call an instance of rule $1(2,3,4,5,6$ or 7$)$ an instance of an axiom-rule $(\rightarrow$ right-rule, $\omega$-rule, $\rightarrow$ left-rule, $\forall$ left-rule, cut rule or falsum rule).

Lemma 1. 1. $F \vdash_{r}^{\alpha} \Gamma \supset C \quad \& \quad \Gamma \subseteq \Gamma^{\prime} \& \quad \alpha \leq \beta \quad \& \quad r \leq r^{\prime} \quad \& \quad F \leq$ $G \& m c(\beta) \leq G(0) \Longrightarrow G \vdash_{r^{\prime}}^{\beta} \Gamma^{\prime} \supset C$.

2. $F \vdash_{r}^{\alpha} A, \Gamma \supset C \& A$ is a literal $\& \mathbb{N} \models A \Longrightarrow F \vdash_{r}^{\alpha} \Gamma \supset C$

3. $F \vdash_{r}^{\alpha} \Gamma \supset A \& A$ is a literal \& $\mathbb{N}=\neg A \Longrightarrow F \vdash_{r}^{\alpha} \Gamma \supset C$.

Proof. By induction on $\alpha$.

Lemma 2 (Inversion). 1. $F \vdash_{r}^{\alpha} \Gamma \supset A \rightarrow B \Longrightarrow F \vdash_{r}^{\alpha} \Gamma, A \supset B$.

2. $F \vdash_{r}^{\alpha} \Gamma \supset \forall x A(x) \Longrightarrow F[m] \vdash_{r}^{\alpha} \Gamma \supset A(\underline{m})$ for all $m \in \mathbb{N}$.

Proof. By induction on $\alpha$. 
With $\alpha \# \beta$ we denote the commutative natural (or Hessenberg) sum of $\alpha$ and $\beta$. Thus $\alpha \# 0=0 \# \alpha=\alpha$ and if $\alpha=\omega^{\alpha_{1}}+\cdots+\omega^{\alpha_{m}} \geq \alpha_{1} \ldots \geq \alpha_{m}$ and $\beta=\omega^{\alpha_{m+1}}+\cdots+\omega^{\alpha_{m+n}} \geq \alpha_{m+1} \geq \ldots \geq \alpha_{m+n}$ then $\alpha \# \beta=\omega^{\alpha_{\pi(1)}}+$ $\cdots+\omega^{\alpha_{p i(m+n)}}$ where $\pi:\{1, \ldots, m+n\} \rightarrow\{1, \ldots, m+n\}$ is a permutation such that $\alpha_{\pi(1)} \geq \ldots \geq \alpha_{\pi(m+n)}$. For given operators $F, G$ let $R(F, G)$ be defined by $R(F, G):=F \circ G+G+F$ where operations are defined pointwise and $\circ$ denotes composition. (What is nice with this approach is that it can be adapted easily to other formulations of $Z^{\infty}$ as well. A very general definition of $R$ would be its symmetric version $R(F, G):=G \circ F+F+F \circ G+G$ which will be appropriate for most other choices of cut elimination procedures. This paper's approach is not affected by such small modifications.)

Lemma 3. If $F \vdash_{m}^{\alpha} \Gamma \supset D$ and $G \vdash_{m}^{\beta} \Gamma, D \supset C$ and $r k(D) \leq m$ then

$$
R(F, G) \vdash_{m}^{\alpha \# \beta \# \beta} \Gamma \supset C .
$$

Proof. By induction on $\beta$. (We follow the lecture notes 'Logic II' of Wilfried Buchholz. In addition we assign appropriate control operators to the derivations.) Assume first that the last inference was $(\rightarrow l)$ with $A \rightarrow B \in D, \Gamma$ and premises

$$
G \vdash_{m}^{\beta_{0}} D, \Gamma \supset A
$$

and

$$
G \vdash_{m}^{\beta_{0}} \Gamma, B, D \supset C
$$

where $\beta_{0}<\beta$. The induction hypothesis yields

$$
R(F, G) \vdash_{m}^{\alpha \# \beta_{0} \# \beta_{0}} \Gamma \supset A
$$

and

$$
R(F, G) \vdash_{m}^{\alpha \# \beta_{0} \# \beta_{0}} \Gamma, B \supset C .
$$

If $A \rightarrow B \in \Gamma$, then the assertion follows from (2) and (3) by $(\rightarrow l)$. Assume now that $D=A \rightarrow B$. The inversion lemma yields

$$
F \vdash_{m}^{\alpha} \Gamma, A \supset B
$$

By (2), (4) and assertion 1 of Lemma 1 we obtain

$$
R(F, G) \vdash_{m}^{\alpha \# \beta \# \beta_{0}} \Gamma \supset B
$$

by an application of the cut rule since $r k(A)<r k(D)$ and

$$
\begin{aligned}
& m c\left(\alpha \# \beta_{0} \# \beta\right) \\
\leq & F(0)+G(0)+G(0) \\
\leq & R(F, G)(0) .
\end{aligned}
$$

With (3) and (5) we obtain

$$
R(F, G) \vdash_{m}^{\alpha \# \beta \# \beta} \Gamma \supset C
$$


by another application of the cut rule since $r k(B)<r k(D)$ and $m c(\alpha \# \beta \# \beta) \leq$ $R(F, G)(0)$.

Assume in the critical case that the last inference is an instance of an $\forall$ leftrule with $\forall x A(x) \in D, \Gamma$ and

$$
G \vdash \underset{m}{\beta_{0}} A(\underline{k}), D, \Gamma \supset C
$$

with

$$
k \leq G(0)
$$

for some $\beta_{0}<\beta$. The induction hypothesis yields

$$
R(F, G) \vdash_{m}^{\alpha \# \beta_{0} \# \beta_{0}} A(\underline{k}), \Gamma \supset C
$$

If $\forall x A(x) \in \Gamma$, then the assertion follows from (8) by $(\forall l)$. If $\forall x A(x)=D$, then assertion 2 of Lemma 1 yields

$$
F[k] \vdash_{m}^{\beta_{0}} \Gamma \supset A(\underline{k}) .
$$

A cut with $A(k)$ yields the assertion provided that $F[k] \leq R(F, G)$. Here the composition with $G$ and (7) come to rescue. Indeed by using the weak monotonicity and the inflationarity of the operators we obtain

$$
F[k](l)=F(\max \{k, l\}) \leq F(\max \{G(0), l\}) \leq R(F, G)(l) .
$$

Finally notice that $m c(\alpha \# \beta \# \beta) \leq R(F, G)(0)$.

For a given $F$ let the $\alpha$-th iterate of $R$ applied to $F, R^{\alpha}(F)$, be defined via $R^{0}(F)(l):=F(l)$ and for $\alpha>0$

$$
R^{\alpha}(F)(l):=\max \left\{R\left(R^{\gamma}(F),\left(R^{\gamma}(F)\right)(l): \gamma<\alpha \quad \& m c(\gamma) \leq F(l)\right\} .\right.
$$

If $F$ is a Skolem operator then (via induction on $\alpha$ ) $R^{\alpha}(F)$ is it, too.

Lemma 4. If $l \geq n$, then $R^{\alpha}(F[n])(l) \leq R^{\alpha}(F)(l)$.

Proof. $R^{0}(F[n])(l)=F[n](l)=F(l)=R^{0}(F)(l)$. Moreover for $\alpha>0$ we have

$$
\left.R^{\alpha}(F[n])(l)=R\left(R^{\gamma}(F[n]), R^{\gamma}(F[n])\right)(l)\right)
$$

for some $\gamma<\alpha$ with $m c(\gamma) \leq F[n](l)=F(l)$. Since $R^{\gamma}(F)(l) \geq l \geq n$ the induction hypothesis yields

$$
\begin{aligned}
& R\left(R^{\gamma}(F[n]), R^{\gamma}(F[n])\right)(l) \\
= & R^{\gamma}(F[n])\left(R^{\gamma}(F[n])(l)\right)+R^{\gamma}(F[n])(l) \cdot 2 \\
\leq & R^{\gamma}(F)\left(R^{\gamma}(F)(l)\right)+R^{\gamma}(F)(l) \cdot 2 \\
= & R\left(R^{\gamma}(F),\left(R^{\gamma}(F)\right)(l)\right. \\
\leq & R^{\alpha}(F)(l) .
\end{aligned}
$$


Theorem 1. If $F \vdash_{m+1}^{\alpha} \Gamma \supset C$, then $\left.R^{\alpha}(F)\right) \vdash_{m}^{\omega^{\alpha}} \Gamma \supset C$.

Proof. (Again we follow the lecture notes 'Logic II' of Wilfried Buchholz. In addition we assign appropriate control operators to the derivations.) There are two critical cases: (cut) and $(\forall r)$. Assume that the last inference was a cut with cut formula $D$. Then we have the premises

$$
F \vdash \vdash_{m+1}^{\alpha_{0}} \Gamma \supset D
$$

and

$$
F \vdash_{m+1}^{\alpha_{0}} \Gamma, D \supset C,
$$

where

$$
m c\left(\alpha_{0}\right) \leq F(0) .
$$

The induction hypothesis yields

$$
R^{\alpha_{0}}(F) \vdash_{m}^{\omega^{\alpha}} \Gamma \supset D
$$

and

$$
R^{\alpha_{0}}(F) \vdash_{m}^{\omega^{\alpha}} \Gamma, D \supset C .
$$

The reduction lemma yields

$$
R\left(R^{\alpha_{0}}(F), R^{\alpha_{0}}(F)\right) \vdash_{m}^{\omega^{\alpha_{0}} \# \omega^{\alpha_{0}} \# \omega^{\alpha_{0}}} \Gamma \supset C .
$$

The claim follows if $R\left(R^{\alpha_{0}}(F), R^{\alpha_{0}}(F)(l) \leq R^{\alpha}(F)(l)\right.$ holds for all $l$. But this follows immediately from (10) and the definition of $R^{\alpha}$.

The remaining critical case is an instance of the $\forall$ right rule and we encounter some intrinsic difficulty, which luckily can be resolved by Lemma 4 . Assume that $F \vdash_{m+1}^{\alpha} \Gamma \supset \forall x A(x)$ has been inferred from $F[n] \vdash_{m+1}^{\alpha_{n}} \Gamma \supset A(\underline{n})$ where

$$
m c\left(\alpha_{n}\right) \leq F[n](0)=F(n) .
$$

The induction hypothesis yields

$$
R^{\alpha_{n}}(F[n]) \vdash_{m}^{\omega^{\alpha_{n}}} \Gamma \supset A(\underline{n}) .
$$

Then

$$
\left(R^{\alpha}(F)\right)[n] \vdash_{m}^{\omega^{\alpha} n} \Gamma \supset A(\underline{n})
$$

and

$$
R^{\alpha}(F) \vdash_{m}^{\omega^{\alpha}} \Gamma \supset \forall x A(x),
$$

provided that $R^{\alpha_{n}}(F[n])(l) \leq\left(R^{\alpha}(F)\right)[n](l)$ holds for all $l$. If $l \geq n$, then Lemma 4 yields

$$
R^{\alpha_{n}}(F[n])(l) \leq R^{\alpha_{n}}(F)(l) \leq R^{\alpha}(F)(l)
$$

since $m c\left(\alpha_{n}\right) \leq F(n) \leq F(l)$ by (11). If $l<n$, then the Lemma 4 yields

$$
R^{\alpha_{n}}(F[n])(l) \leq R^{\alpha_{n}}(F[n])(n) \leq R^{\alpha_{n}}(F)(n) \leq R^{\alpha}(F)(n) \leq\left(R^{\alpha}(F)\right)[n](l)
$$

again by (11).

All other non-critical inferences are taken care of straightforwardly by the induction hypothesis. 
Lemma 5. Assume that $A$ is a negated equation. If $F \vdash_{0}^{\alpha} \forall x A(x) \supset \perp$ then there is a $m \leq F(0)$ such that $\mathbb{N} \mid=\neg A[m]$

Proof. By induction on $\alpha$. Since the derivation is cut free the last rule was an instance of the $\forall$ left rule or the falsum rule. Assume first that the last inference rule was an instance of the $\forall$ left rule. Then there is an $\alpha_{0}<\alpha$ and a $k \leq F(0)$ such that $F \vdash_{0}^{\alpha_{0}} A(\underline{k}), \forall x A(x) \supset \perp$. If $\mathbb{N} \models \neg A[k]$, then we are done. Otherwise $\mathbb{N} \models A[k]$ and by assertion 2 of Lemma 1 we obtain $F \vdash_{0}^{\alpha_{0}} \forall x A(x) \supset \perp$. Now the induction hypothesis yields that there is an $m \leq F(0)$ such that $\mathbb{N} \models \neg A[m]$.

Finally assume that the last inference rule was the falsum rule. Then there is an $\alpha_{0}<\alpha$ such that $F \vdash_{0}^{\alpha_{0}} \neg \perp, \forall x A \supset \perp$. Then assertion 2 of Lemma 1 yields $F \vdash_{0}^{\alpha_{0}} \forall x A \supset \perp$ and the claim follows from the induction hypothesis.

Theorem 2 (Collapsing Theorem). Assume that $A$ is an equation. Assume that $F \vdash_{0}^{\alpha} \supset(\forall x)(\exists y) A(x, y)$. Then for all $m$ there exists an $n \leq F(m)$ such that $\mathbb{N}=A[m, n]$.

Proof. $F \vdash_{0}^{\alpha} \supset(\forall x)(\exists y) A(x, y)$ yields $F[m] \vdash_{0}^{\alpha} \supset(\exists y) A(\underline{m}, y)$. Thus $F[m] \vdash_{0}^{\alpha}$ $(\forall y) \neg A(\underline{m}, y) \supset \perp$. Hence by Lemma 5 there is an $n \leq F[m](0)=F(m)$ such that $\mathbb{N} \models A[m, n]$.

We write $A \sim A^{\prime}$ if there are a $L_{Z}$-formula $B\left(x_{1}, \ldots, x_{n}\right)$ with pairwise distinct variables $x_{1}, \ldots, x_{n}$ and closed $L_{Z}$-terms $t_{1}, s_{1}, \ldots, t_{n}, s_{n}$ such that $\operatorname{val}\left(t_{i}\right)=$ $\operatorname{val}\left(s_{i}\right)$ for $i=1, \ldots, n$ and $A=B\left(t_{1}, \ldots, t_{n}\right), A^{\prime}=B\left(s_{1}, \ldots, s_{n}\right)$.

Lemma 6 (Tautology Lemma). If $C \sim C^{\prime}$ then $\lambda x .(2 \cdot r k(C)+x) \vdash_{0}^{2 \cdot r k(C)}$ $C \supset C^{\prime}$

Proof. By induction on $r k(C)$.

Lemma 7. If $Z \vdash C$ and $F V(C) \subseteq\left\{x_{1}, \ldots, x_{n}\right\}$, then there exists a primitive recursive operator $F$ and $k, r$ such that $F\left[m_{1}, \ldots, m_{n}\right] \vdash_{r}^{\omega+k} \supset C\left[\underline{m_{1}}, \ldots, \underline{m_{n}}\right]$.

Proof. By induction on $Z \vdash C$. We only treat the induction scheme. Assume that $C$ has the form $F(0) \&(\forall x)[F(x) \rightarrow F(S(x))] \rightarrow \forall x F(x)$ and assume for simplicity that $C$ is closed. We prove

$$
\lambda x(2 \cdot r k(F)+2 \cdot x)[n] \vdash_{0}^{2 \cdot r k(F)+2 \cdot n} F(0), \forall x(F(x) \rightarrow F(S(x))) \supset F(\underline{n})
$$

by induction on $n$. If $n=0$ then the assertion follows from the Tautology Lemma 6. Let $H(x)=2 \cdot r k(F)+2 \cdot x$. Assume that

$$
H[n] \vdash_{0}^{2 \cdot r k(F)+2 \cdot n} F(0), \forall x(F(x) \rightarrow F(S(x))) \supset F(\underline{n}) .
$$

Let $G(x)=2 \cdot r k(C)+x$. The Tautology Lemma 6 yields

$$
G \vdash_{0}^{2 \cdot r k(F)} F(S(\underline{n})) \supset F(S(\underline{n})) .
$$


Therefore

$H[n+1] \vdash_{0}^{2 \cdot r k(F)+2 \cdot n+1} F(0), F(\underline{n}) \rightarrow F(S(\underline{n})), \forall x(F(x) \rightarrow F(S(x))) \supset F(S(\underline{n}))$.

An application of the $\forall$ left rule yields the assertion.

As a useful abbreviation we define $\omega_{k}(\alpha)$ recursively as follows. $\omega_{0}(\alpha):=\alpha$ and $\omega_{k+1}(\alpha):=\omega^{\omega_{k}(\alpha)}$.

Let us assume that $T$ is a standard primitive recursive Kleene predicate for the unary partial recursive functions. Moreover if $\mathbb{N}=(\forall x)(\exists y) T(\underline{e}, x, y)$, then $\varphi_{e}$ denotes the function $m \mapsto U(\mu n: \mathbb{N} \models T[e, m, n])$.

Corollary 1. Assume that PA $\vdash(\forall x)(\exists y) T(\underline{e}, x, y)$. Then there is an $\alpha<\varepsilon_{0}$ such that $\varphi_{e}$ is $\alpha$-recursive.

Proof. Assume PA $\vdash(\forall x)(\exists y) T(\underline{e}, x, y)$. Then $Z \vdash(\forall x)(\exists y) T(\underline{e}, x, y)$. Hence there exists a primitive recursive operator $F$ and $k, r$ such that

$$
F \vdash_{r}^{\omega+k} \supset(\forall x)(\exists y) T(\underline{e}, x, y) .
$$

By applying the reduction theorem we obtain an operator $G$ defined by $\omega_{r}(\omega+k)$ recursion from $F$ such that

$$
G \vdash_{0}^{\omega_{r}(\omega+k)} \supset(\forall x)(\exists y) T(\underline{e}, x, y) .
$$

But then $\varphi_{e}$ is primitive recursive in $G$ by the collapsing theorem.

To obtain a complete classification of the provably recursive functions, we relate the operators with the classical Hardy hierarchy. This hierarchy is defined with respect to a system of standard fundamental sequences. This system is defined as follows. If $\alpha=\omega^{\alpha_{1}}+\cdots+\omega^{\alpha_{n}+1}$ in normal form, then

$$
\alpha[x]:=\omega^{\alpha_{1}}+\cdots+\omega^{\alpha_{n}} \cdot(x+1) .
$$

If $\alpha=\omega^{\alpha_{1}}+\cdots+\omega^{\alpha_{n}}$ in normal form with $\alpha_{n} \in \operatorname{Lim}$, then

$$
\alpha[x]:=\omega^{\alpha_{1}}+\cdots+\omega^{\alpha_{n}[x]} .
$$

If $\alpha=\beta+1$, then $\alpha[x]:=\beta$ and $0[x]:=0$. Finally put $\varepsilon_{0}[x]:=\omega_{x}(1)$. Define the Hardy hierarchy $\left.\left(H_{\alpha}\right)_{\alpha \leq \varepsilon_{0}}\right)$ as follows:

1. $H_{0}(x)=x$,

2. $H_{\alpha}(x)=H_{\alpha[x]}(x+1)$ for $\alpha>0$.

Our goal is to show that every provably recursive function of PA is primitive recursive in $H_{\alpha}$ for some $\alpha<\varepsilon_{0}$. Moreover each $H_{\alpha}$ is provably recursive in PA.

Theorem 3. For any $\alpha<\varepsilon_{0} \mathrm{PA} \vdash(\forall x)(\exists z)\left[H_{\alpha}(x)=z\right]$. 
Proof. The proof follows by formalizing the computation of $H_{\alpha}(x)$. A convenient choice for representing $\varepsilon_{0}$ in PA is e.g. as follows. Let $\left(p_{i}\right)_{i \geq 1}$ be an enumeration of the prime numbers. Let $\operatorname{ind}\left(p_{i}\right):=i$. Let $(m, n)$ denotes the greatest common divisor of $m$ and $n$. Put

$$
\begin{aligned}
m \prec n \Longleftrightarrow & m \neq n \&[m=1 \vee n=0 \vee \\
& {\left.\left[\left(\forall p \mid \frac{m}{(m, n)}\right)\left(\exists p^{\prime} \mid \frac{n}{(m, n)}\right) \operatorname{ind}(p) \prec \operatorname{ind}\left(p^{\prime}\right)\right]\right] }
\end{aligned}
$$

where $p, p^{\prime}$ range over primes. Then 0 has order type $\varepsilon_{0}$ whereas 1 has order type 0 . The $n$-th natural number is coded by $2^{n}, \omega$ is coded by 3 and $\omega^{\omega}$ by 5 etc. It is well known that PA proves for any $m \neq 0$ the following scheme for any $L_{\mathrm{PA}}$ formula $F$ :

$$
\forall x(\forall y \prec x F(y) \rightarrow F(x)) \Longrightarrow \forall x \prec \underline{m} F(x)
$$

We therefore introduce a formula $H(a, x, y, z)$ such that (provably within PA) $H(a, x, y, z)$ holds iff $y$ is a computation tree for $H_{\alpha}(x)$ giving $z$ as value of $H_{\alpha}(x)$ when $a$ codes $\alpha$. The idea is to trace the recursive definition of $H_{\alpha}(x)$ backwards to $H_{0}(z)$ and then to read off the result $z$ at that moment by looking at the input value $z$. Assume that $a, x \mapsto a[x]$ is a fixed primitive recursive assignment of fundamental sequences to the codes. Then $H(a, x, y, z): \Longleftrightarrow$ $y \in \operatorname{Seq} \&\left(\forall i<\operatorname{lh}(y)\left[\operatorname{lh}\left((y)_{i}\right)=3\right.\right.$

$\left.\left.\left.\& i=0 \rightarrow(\exists v \leq y)\left[(y)_{i}=\langle 1, v, v\rangle\right]\right)\right]\right)$

$\& i=\operatorname{lh}(y)-1 \rightarrow(y)_{i}=\langle a, x, z\rangle$

$\& i+1<\operatorname{lh}(y) \rightarrow(\forall u, v, w)\left[(y)_{i+1}=\langle u, v, w\rangle \rightarrow(y)_{i}=\langle u[v], v+1, w\rangle\right]$.

Then we obtain within PA the equivalence

$$
H(a[x], x+1, y, z) \leftrightarrow H(a, x, y \star\langle a, x, z\rangle, z) .
$$

By induction up to any fixed $\alpha$ with code $\underline{a}$ one proves within PA using (12)

$$
b \preceq \underline{a} \quad \& H(b, x, y, z) \quad \& \quad H\left(b, x, y^{\prime}, z^{\prime}\right) \rightarrow y=y^{\prime} \quad \& \quad z=z^{\prime} .
$$

Moreover by induction up to any fixed $\alpha$ with code $a$ one proves within PA using (12)

$$
b \preceq \underline{a} \rightarrow(\forall x)(\exists y)(\exists z)[H(b, x, y, z)] .
$$

Indeed, assume $b \preceq \underline{a}$ and $b \neq 1$. Assume $x$ is given. Then $b[x] \prec b$. By the induction hypothesis there exist $y^{\prime}$ and $z^{\prime}$ such that $H\left(b[x], x+1, y^{\prime}, z^{\prime}\right)$. Put $y:=y^{\prime} \star\left\langle b, x, z^{\prime}\right\rangle$ and $z:=z^{\prime}$.

Thus we may define $H_{\alpha}(x)=z$ via the formula $(\exists y) H(\underline{a}, x, y, z)$ where $a$ is the code for $\alpha$. Then PA proves $(\forall x)(\exists z) H_{\alpha}(x)=z$.

We are left with showing that our recursive operators can be majorized by appropriate Hardy functions. For this purpose some elementary theory of the Hardy functions has to be developed. Our presentation follows [8] but is entirely self-contained and so we hope that this proves again useful for teaching purposes. 
Lemma 8. 1. $\alpha \in \operatorname{Lim} \Longrightarrow \alpha[n]<\alpha[n+1] \& \alpha=\sup \{\alpha[n]: n<\omega\}$.

2. $\alpha[n]<\beta<\alpha \Longrightarrow \alpha[n] \leq \beta[0]$.

3. $H_{\beta}(n)<H_{\beta}(n+1)$.

4. $\alpha[m]<\beta<\alpha \Longrightarrow H_{\alpha[m]}(n+1) \leq H_{\beta}(n)$.

5. $\beta<\alpha \in \operatorname{Lim} \& m c(\beta) \leq n \Longrightarrow \beta<\alpha[n]$.

6. $\beta<\alpha \& m c(\beta) \leq n \Longrightarrow H_{\beta}(n)<H_{\alpha}(n)$.

Proof. Assertion 1 follows by induction on $\alpha$. Assertion 2 follows by induction on $\alpha$. Assertions 3 and 4 follow by simultaneous induction on $\beta$. Assertion 5 follows by induction on $\alpha$. Assume that $\alpha=\omega^{\alpha_{1}}+\alpha_{2}$ and $\beta=\omega^{\beta_{1}}+\beta_{2}$ (in normal form). If $\alpha_{1}<\beta_{1}$ and $\beta_{2}>0$, then $\alpha<\omega^{\beta_{1}} \leq \omega^{\beta_{1}}+\beta_{2}[n]=\beta[n]$. If $\alpha_{1}=\beta_{1}$, then $\alpha_{2}<\beta_{2} \in \operatorname{Lim}$ and the induction hypothesis yields $\alpha_{2}<\beta_{2}[n]$ thus $\alpha<\beta[n]$. In the critical case is $\alpha_{1}<\beta_{1}$ and $\beta_{2}=0$. If $\beta_{1} \in \mathrm{Lim}$, then the induction hypothesis yields $\alpha_{1}<\beta_{1}[n]$ hence $\alpha<\omega^{\beta_{1}[n]}=\beta[n]$. Thus assume finally that $\beta_{1}=\beta^{\prime}+1$. If $\alpha<\beta^{\prime}$, then $\alpha<\omega^{\beta^{\prime}} \leq \beta[n]$. Thus we may assume that $\alpha_{1}=\beta^{\prime}$. Write $\alpha=\omega^{\alpha_{1}} \cdot m+\alpha_{3}$ with $\alpha_{3}<\omega^{\alpha_{1}}$. Then $m \leq m c(\alpha) \leq n$. Thus $\alpha<\omega^{\alpha_{1}} \cdot n+\omega^{\alpha_{1}}=\beta[n]$. Assertion 6 follows by induction on $\alpha$ using assertion 5 .

We write $N F(\alpha, \beta)$ if $\alpha=0$ or $\beta=0$ or $\alpha=\omega^{\alpha_{1}}+\cdots+\omega^{\alpha_{m}}$ in normal form and $\beta=\omega^{\beta_{1}}+\cdots+\omega^{\beta_{n}}$ in normal form and $\alpha_{n} \geq \beta_{0}$. Thus $\alpha+\beta$ will be a natural sum in this case.

Lemma 9. $\quad 1 . \beta>0 \quad \& \quad N F(\alpha, \beta) \Longrightarrow(\alpha+\beta)[n]=\alpha+\beta[n]$ and $N F(\alpha, \beta[n])$.

2. $N F(\alpha, \beta) \Longrightarrow H_{\alpha+\beta}=H_{\alpha} \circ H_{\beta}$.

3. $H_{\omega^{\alpha+1}}(n)=H_{\omega^{\alpha}}^{(n+1)}(n+1)$ and $H_{\omega^{\lambda}}(n)=H_{\omega^{\lambda}[n]}(n+1)$ if $\lambda \in \operatorname{Lim}$.

4. $H_{\alpha} \leq H_{\omega^{\alpha}}$.

5. $H_{\alpha \# \beta}(n) \geq H_{\alpha}(n)$.

6. $m c(\alpha[l]) \leq \max \{m c(\alpha), l+1\}$

7. $m c(\alpha) \leq 1+m c(\alpha[l])$

8. $H_{\alpha}(l) \geq l+m c(\alpha)$.

9. $H_{\alpha}(k)+H_{\beta}(l)+m \leq H_{\alpha}\left(k+H_{\beta}(l)+m\right)$.

Proof. Assertions 1 and 2 follow by induction on $\beta$. Assertion 3 follows from assertion 2. Assertion 4 follows by induction on $\alpha$. Assertion 5 follows from assertion 2. Assertions 6 and 7 follow by induction on $\alpha$. Assertion 8 follows by induction on $\alpha$ and 7 . Assertion 9 follows from the strict monotonicity of $H_{\alpha}$. 
Theorem 4. Assume that $F: \mathbb{N} \rightarrow \mathbb{N}$ is a Skolem operator, i.e $F$ is weakly increasing and inflationary. Assume that $F \leq H_{\alpha}$ for some $\alpha<\varepsilon_{0}$. Then $R^{\beta}(F) \leq H_{\omega^{\alpha \# \beta+1}+8}$.

Proof. We prove that $R^{\beta}(F)(l) \leq H_{\omega^{\alpha \# \beta+1}}(l)$ for all $l \geq 8$. If $\beta=0$, then the claim follows from

$$
F(l) \leq H_{\alpha}(l) \leq H_{\alpha+1}(l) \leq H_{\omega^{\alpha+1}}(l) .
$$

Assume that $\beta>0$ Then $R^{\beta}(F)(l)=R\left(R^{\gamma}(F), R^{\gamma}(F)\right)(l)$ for some $\gamma<\beta$ with $m c(\gamma) \leq F(l) \leq H_{\alpha}(l)$. The induction hypothesis yields for $l \geq 8$ (with assertion 9 from Lemma 9, assertion 6 from Lemma 8 and assertions 4 and 5 from Lemma 9):

$$
\begin{aligned}
R\left(R^{\gamma}(F), R^{\gamma}(F)\right)(l) & \leq R\left(H_{\omega \gamma \# \alpha+1}, H_{\omega \gamma \# \alpha+1}\right)(l) \\
& \leq H_{\omega \gamma \# \alpha+1}\left(H_{\omega \gamma \# \alpha+1}(l)\right)+H_{\omega \gamma \# \alpha+1}(l) \cdot 2 \\
& \leq H_{\omega \gamma \# \alpha+1}+\omega^{\gamma \# \alpha+1}(l)+H_{\omega \gamma \# \alpha+1}(l) \cdot 2 \\
& \leq H_{\omega^{\gamma \# \alpha+1.2}}\left(l+2 \cdot H_{\omega^{\gamma \# \alpha+1}}(l)\right) \\
& \leq H_{\omega^{\gamma \# \alpha+1} \cdot 2}\left(H_{\omega \gamma \# \alpha+1}\left(H_{\omega^{\gamma \# \alpha+1}}(3 \cdot l)\right)\right) \\
& \leq H_{\omega \gamma \# \alpha+1.4}(l \cdot 3) \\
& \leq H_{\omega^{\gamma \# \alpha+1} \cdot 4}\left(H_{\alpha}(l) \cdot 3\right) \\
& \leq H_{\omega^{\beta \# \alpha} \cdot 4}\left(H_{\alpha}(l) \cdot 3\right) \\
& \leq H_{\omega^{\beta \# \alpha} \cdot 7}(l) \\
& \leq H_{\omega^{\beta \# \alpha+1}}(l) .
\end{aligned}
$$

Corollary 2. Assume that PA $\vdash(\forall x)(\exists y) T(e, x, y)$. Then there is an $\alpha<\varepsilon_{0}$ such that $\varphi_{e}$ is primitive recursive in and bounded by $H_{\alpha}$.

Corollary 3. PA $\nvdash(\forall x)(\exists y)\left[H_{\varepsilon_{0}}(x)=y\right]$.

Using the usual identification of hydras with ordinals below $\varepsilon_{0}$ we obtain the following result on the termination of the hydra game.

Corollary 4 (PA-unprovability of the termination of the hydra game). $\mathrm{PA} \nvdash\left(\forall \alpha<\varepsilon_{0}\right)(\exists k)[\alpha[0][1] \ldots[k]=0]$.

Using Cichon's 1983 proof from [9] we can now also quickly obtain the independence result for the termination of the Goodstein sequences.

Results of this type may be used to start investigations on phase transitions for independence results ${ }^{2}$ (see, for example, [24] for a recent survey) but we quit at this point.

\footnotetext{
${ }^{2}$ This was the topic of a seminar by the author in Utrecht which followed his proof and recursion theory lecture.
} 


\section{References}

[1] W. Ackermann: Zur Widerspruchsfreiheit der reinen Zahlentheorie, Mathematische Annalen 117, 1940.

[2] J. Avigad and R. Sommer. A model-theoretic approach to ordinal analysis. Bull. Symbolic Logic 3 (1997), no. 1, 17-52.

[3] B. Blankertz and A. Weiermann: How to characterize provably total functions by the Buchholz operator method. Springer Lecture Notes in Logic 6 (1996), 205-213.

[4] W. Buchholz: Notation systems for infinitary derivations. Archive for Mathematical Logic 30 5/6 (1991), 277-296.

[5] W. Buchholz: A simplified version of local predicativity. Proof Theory: A Selection of Papers from the Leeds Proof Theory Meeting 1990, P. Aczel, H. Simmons, S.S. Wainer, editors. Cambridge University Press (1992), 115148.

[6] W. Buchholz: Lectures Notes Logic I, II and proof theory. Online available under: http://www.mathematik.uni-muenchen.de/ buchholz/

[7] W. Buchholz und S.S. Wainer: Provably computable functions and the fast growing hierarchy. Logic and Combinatorics. Contemporary Mathematics 65, American Mathematical Society (1987), 179-198.

[8] W. Buchholz, A. Cichon and A. Weiermann: A uniform approach to fundamental sequences and hierarchies. Mathematical Logic Quarterly 40 (1994), 273-286.

[9] E.A. Cichon. A short proof of two recently discovered independence results using recursion theoretic methods. Proceedings of the AMS 87 (1983) 704706.

[10] M. Fairtlough and S.S. Wainer: Hierarchies of provably recursive functions. Handbook of proof theory, 149-207, Stud. Logic Found. Math., 137, NorthHolland, Amsterdam, 1998.

[11] H. Friedman and M. Sheard: Elementary descent recursion and proof theory. Annals of Pure and Applied Logic 71 (1995), 1-45.

[12] K. Gödel: Über eine bisher noch nicht benützte Erweiterung des finiten Standpunkts. Dialectica 12 (1958), 280-287.

[13] P. Hájek and P. Pudlák: Metamathematics of First-Order Arithmetic, Springer (1993).

[14] G. Kreisel: On the interpretation of non-finitist proofs II. The Journal of Symbolic Logic 17 (1952), 43-58. 
[15] W. Pohlers: Proof Theory. An Introduction. Lecture Notes in Mathematics 1407 (1989). Springer, Berlin.

[16] W. Pohlers. Subsystems of set theory and second order number theory. Handbook of proof theory, 209-335, Stud. Logic Found. Math., 137, NorthHolland, Amsterdam, 1998.

[17] H. E. Rose: Subrecursion: Functions and Hierarchies. Oxford University Press 1984.

[18] K. Schütte: Proof Theory. Springer, Berlin 1977.

[19] H. Schwichtenberg: Eine Klassifikation der $\varepsilon_{0}$-rekursiven Funktionen. Zeitschrift für Mathematische Logik und Grundlagen der Mathematik (1971), 61-74.

[20] H. Schwichtenberg: Proof theory: Some applications of cut-elimination. Handbook of Mathematical Logic, J. Barwise (ed.), North-Holland (1977), 867-895.

[21] G. Takeuti: Proof Theory, 2nd edition. North-Holland, Amsterdam 1987.

[22] S.S. Wainer: A classification of the ordinal recursive functions. Archiv für Mathematische Logik 13 (1970), 136-153.

[23] A. Weiermann: How to characterize provably total functions by local predicativity. The Journal of Symbolic Logic.

[24] A. Weiermann: Analytic combinatorics, proof-theoretic ordinals, and phase transitions for independence results. Ann. Pure Appl. Logic 136 (2005), no. $1-2,189-218$. 